\title{
Baicalin Ameliorates Experimental Liver Cholestasis in Mice by Modulation of Oxidative Stress, Inflammation, and NRF2 Transcription Factor
}

\author{
Kezhen Shen, ${ }^{1,2}$ Xiaowen Feng, ${ }^{1,2}$ Hao Pan, ${ }^{3}$ Feng Zhang, ${ }^{1,2}$ Haiyang Xie, ${ }^{1,2}$ and \\ Shusen Zheng ${ }^{1,2,4}$ \\ ${ }^{1}$ Key Laboratory of Combined Multi-Organ Transplantation, Ministry of Public Health, the First Affiliated Hospital, \\ College of Medicine, Zhejiang University, Hangzhou, Zhejiang 310003, China \\ ${ }^{2}$ Collaborative Innovation Center for Diagnosis and Treatment of Infectious Diseases, the First Affiliated Hospital, \\ College of Medicine, Zhejiang University, Hangzhou, Zhejiang 310003, China \\ ${ }^{3}$ Department of Urology, the First Affiliated Hospital, College of Medicine, Zhejiang University, Hangzhou, Zhejiang 310003, China \\ ${ }^{4}$ Division of Hepatobiliary and Pancreatic Surgery, the First Affiliated Hospital, Zhejiang University School of Medicine, Hangzhou, \\ Zhejiang 310003, China
}

Correspondence should be addressed to Shusen Zheng; shusenzheng@zju.edu.cn

Received 25 March 2017; Revised 18 May 2017; Accepted 28 May 2017; Published 5 July 2017

Academic Editor: Aditya Sen

Copyright (C) 2017 Kezhen Shen et al. This is an open access article distributed under the Creative Commons Attribution License, which permits unrestricted use, distribution, and reproduction in any medium, provided the original work is properly cited.

\begin{abstract}
Experimental cholestatic liver fibrosis was performed by bile duct ligation (BDL) in mice, and significant liver injury was observed in 15 days. Administration of baicalin in mice significantly ameliorates liver fibrosis. Experimental cholestatic liver fibrosis was associated with induced gene expression of fibrotic markers such as collagen I, fibronectin, alpha smooth muscle actin (SMA), and connective tissue growth factor (CTGF); increased inflammatory cytokines (TNF $\alpha$, MIP1 $\alpha$, IL1 $\beta$, and MIP2); increased oxidative stress and reactive oxygen species- (ROS-) inducing enzymes (NOX2 and iNOS); dysfunctional mitochondrial electron chain complexes; and apoptotic/necrotic cell death markers (DNA fragmentation, caspase 3 activity, and PARP activity). Baicalin administration on alternate day reduced fibrosis along with profibrotic gene expression, proinflammatory cytokines, oxidative stress, and cell death whereas improving the function of mitochondrial electron transport chain. We observed baicalin enhanced NRF2 activation by nuclear translocation and induced its target genes HO-1 and GCLM, thus enhancing antioxidant defense. Interplay of oxidative stress/inflammation and NRF2 were key players for baicalin-mediated protection. Stellate cell activation is crucial for initiation of fibrosis. Baicalin alleviated stellate cell activation and modulated TIMP1, SMA, collagen 1, and fibronectin in vitro. This study indicates that baicalin might be beneficial for reducing inflammation and fibrosis in liver injury models.
\end{abstract}

\section{Introduction}

Chronic liver disease prevalence is increasing globally, and liver fibrosis associated with such chronic liver disease is an important cause of morbidity and mortality in the world [1]. Due to repetitive injury and inflammation in chronic liver disease, significant extracellular matrix is deposited and causes severe liver damage and organ failure. This liver fibrosis can also lead to cirrhosis and hepatocellular carcinoma [1]. Chronic cholestatic liver disease is also observed after liver transplantation [2]. Unfortunately limited antifibrotic therapies are available to treat patients with liver fibrosis [3, 4]. Many plant-derived flavonoids including baicalin show antifibrotic properties in the liver of animal models, but the mechanistic studies are limited.

The proposed mechanism of liver fibrosis includes oxidative tissue injury, mitochondrial dysregulation, and inflammation, which lead to apoptotic and necrotic cell death $[5,6]$. Liver fibrogenic cells (myofibroblasts), mainly derived from hepatic stellate cells (HSC), play a central role during 


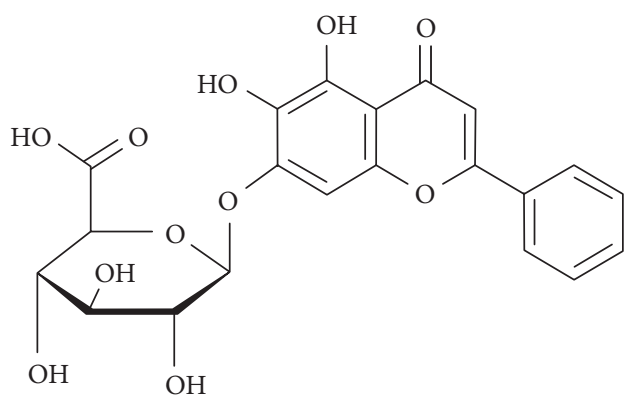

(a)

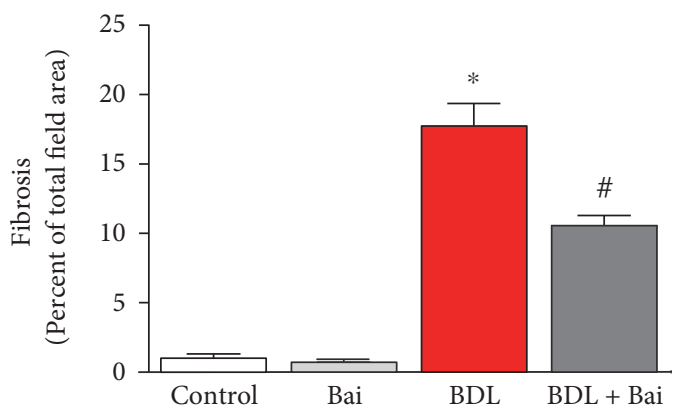

(c)
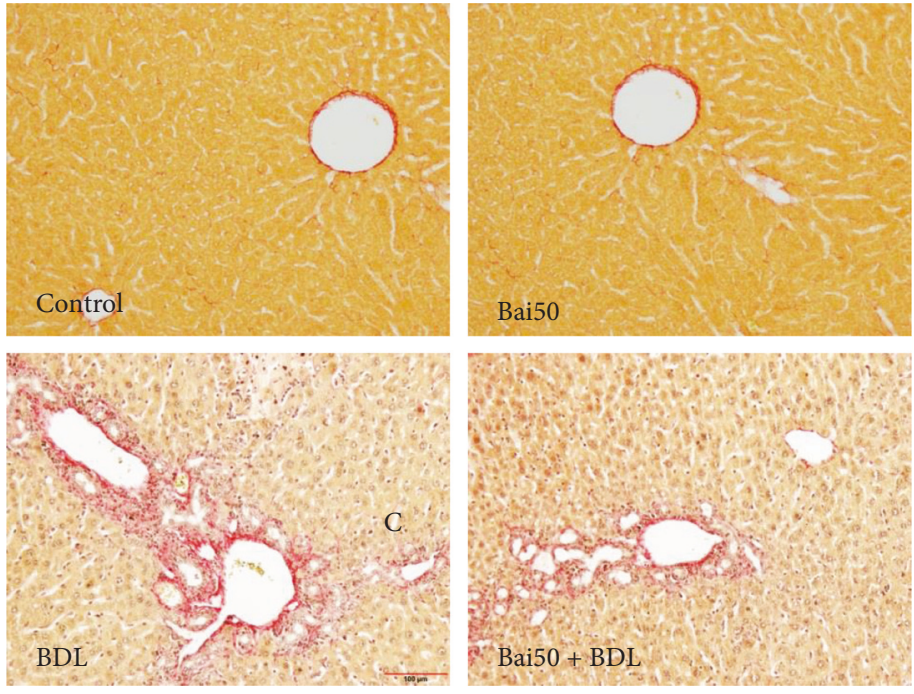

(b)
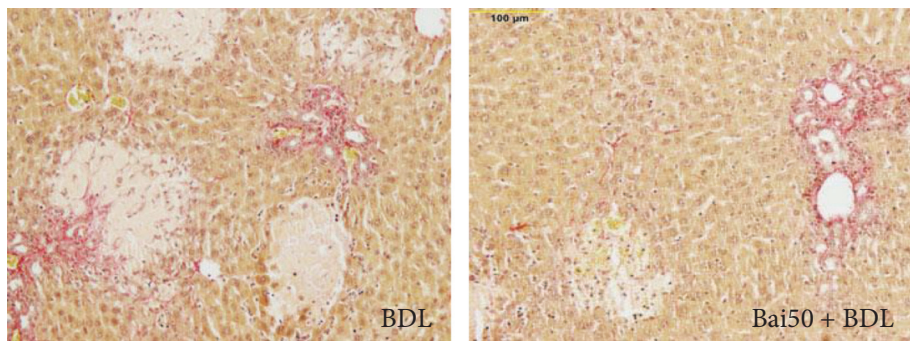

(d)

FIgure 1: Baicalin significantly attenuated BDL-induced liver fibrosis in mice. (a) Chemical structure of baicalin. (b) BDL-induced liver fibrosis as measured by picrosirius red staining. The images were quantified in a blinded manner by ImageJ software (c). BDL resulted in severe liver fibrosis which was attenuated by baicalin treatment. Results are mean \pm S.E.M. $n=6$ for each group. ${ }^{*} p<0.05$ versus control and ${ }^{\#} p<0.05$ versus BDL. (d) Representative images for highlighting necrosis area in BDL and BDL samples pretreated with baicalin.

liver fibrosis [7]. At early stage of tissue injury, all surrounding cells to HSC contribute to its activation. Inflammationmediated hepatocyte cell death and its engulfment by HSC result in a profibrogenic response and also activate Kupffer cells $[8,9]$.

Flavonoids are natural compounds and are of considerable interest in the field of drug discovery as well as food supplement industry [10]. Baicalin is one such flavonoid and derived from the roots of Scutellaria baicalensis Georgi, and these plants are mainly cultivated in China, Siberia, Russia, and Mongolia [11]. The chemical nomenclature (IUPAC) is 5,6-dihydroxy-4-oxo-2-phenyl-4H-chromen-7yl $\beta$-D-glucopyranosiduronic acid (Figure 1(a)). Protective effects of baicalin in many liver disease animal models have been reported. In a rat ischemia/reperfusion injury model, baicalin protects the liver by antioxidant and antiinflammatory properties [12]. In concanavalin A-induced mouse liver injury, baicalin protects by modulating cytokine production [13]. Baicalin attenuates alcoholic liver injury involving sonic hedgehog pathway in addition to its antioxidant and anti-inflammatory properties [14]. In a high-fat diet-induced obesity model of mice, baicalin attenuates liver dysfunction by inhibiting CaMKK/AMPK/ACC pathway and protein kinase $\mathrm{B} / \mathrm{glycogen}$ synthase kinase 3 beta pathway $[15,16]$. Recently, baicalin is shown to protect the carbon tetrachloride-induced liver injury model of the rat by activating PPAR $\gamma$ and inhibiting TGF $\beta 1$ [17]. Here, we demonstrated the protective effect of baicalin in a cholestatic mice model was mediated via NRF2 transcription factor and its downstream target in addition to modulation of oxidative stress-generating enzymes and inflammation.

\section{Materials and Methods}

2.1. Mice Surgeries and Baicalin Treatment. Animal Ethics Review Committees of Zhejiang University approved the animal study protocol. Male inbred C57BL/6 (H2b) mice at 6-8 weeks old were obtained from the Animal Research Institution of Zhejiang Province (Hangzhou). BDL surgeries were performed under anesthesia as described earlier [5]. To determine the effect of baicalin on liver fibrosis, mice received one dose of preoperative (2 hours) IP injection of baicalin and alternate day postoperative IP injection of 
baicalin (50 mg/kg/day) for additional 14 days. For all animal experiments, we used 12 mice/group.

2.2. Liver Fibrosis Marker. Quantitative determination of liver fibrosis was performed with Picrosirius Red Stain kit (Abcam Company Ltd., China) as described earlier [5]. The quantification of collagen staining (red) was analyzed from images using ImageJ software (NIH, USA).

2.3. $m R N A$ Quantification. RNA isolation and reverse transcription were carried out according to the previously published method [5]. Fold change was calculated as described earlier [18]. The gene-specific primers were purchased from Qiagen (USA).

2.4. Mitochondrial Electron Transport Chain Complexes. Activities of mitochondrial electron transport chain complex I, complex II, and complex IV were performed (Mitosciences, USA), and activities were expressed as fold change as described earlier [5]. Activities were determined from total tissue lysates as recommended by the manufacturer.

2.5. Liver Oxidative Stress Markers. Liver 3-NT levels were determined with ELISA kit from Hycult Biotechnology, Cell Sciences, Canton, USA, as described earlier [5].

HNE adducts were determined using the OxiSelect ${ }^{\mathrm{TM}}$ HNE Adduct ELISA Kit according to manufacturer's instruction (Cell Biolabs, Genetimes Technology Inc., Shanghai, China) as described earlier [19].

2.6. Nuclear and Cytoplasmic Fractionation. Nuclear and cytoplasmic fractions were separated from the same set of samples by commercial kit (NE-PER Nuclear and Cytoplasmic Extraction Reagents, Thermo Fisher Scientific, USA).

2.7. Mitochondria Isolation. Mitochondria were isolated using commercial tissue mitochondria isolation kit (Pierce, USA) as described earlier [5].

2.8. Immunoblot Analyses. The protein contents were determined according to the Bradford method. Aliquots of 40 micrograms of protein were analyzed by SDS-PAGE. Western blots were carried out using antibodies for $\mathrm{TNF} \alpha$, GAPDH, NRF2, and lamin A (Abcam Company Ltd., China), Secondary antibodies were purchased from Fazendo Media (Beijing, China).

2.9. Liver PARP Activity. Liver PARP activities were determined using the commercial assay kit (Trevigen Inc.) as described earlier [5]. The quantitative values were expressed as fold change compared to those of the sham control.

2.10. Liver Apoptotic Markers. Caspase 3 activities were measured by using colorimetric caspase 3 activity assay kit (Beyotime Biotech, China) according to the manufacturer's instruction and expressed as fold change. DNA fragment quantifications were performed with the DNA fragmentation kit (Takara, China) according to the manufacturer's protocol.
2.11. Stellate Cell Isolation and Culture. Primary stellate cells from mice were isolated based on our earlier published method [5]. Primary stellate cells were grown for 8 days. Baicalin was added at $20 \mu \mathrm{M}$ every day after replacing with fresh media. RNA was extracted either from fresh culture (day 0 ) or after 8 days (day 8), and real-time PCR was performed after reverse transcription.

2.12. Statistical Analysis. All graphical data were shown as the means \pm SEMs. Multiple comparisons were performed using one-way ANOVA using Graph Pad Prism software (USA). A $p$ value less than 0.05 was considered statistically significant.

\section{Results and Discussions}

3.1. Baicalin Attenuates BDL-Induced Liver Fibrosis in Mice. BDL for 15 days induced significant fibrosis based on picosirius red staining, which stains collagen proteins in parenchymal cells (Figure 1(b)). Treatment with baicalin at $200 \mathrm{mg} / \mathrm{kg}$ dose on alternate day reduced fibrosis. Image quant analysis of the images demonstrated that 17.9-fold increased in fibrotic area was observed due to BDL and baicalin attenuated up to $40.3 \%$ (Figure $1(\mathrm{c})$ ). In addition to fibrosis, significant increase of hepatocyte necrosis was observed and baicalin treatment attenuated those necrosis incidents (Figure 1(d)). To understand the molecular basis of fibrosis, we determined mRNA level of genes associated with fibrosis by real-time PCR. Collagen I, fibronectin, SMA, and CTGF were induced to 7.3-, 6.8-, 7.5-, and 8.3fold, respectively, in BDL model (Figure 2). Alternate day of baicalin treatment attenuated BDL-induced mRNA of collagen I, fibronectin, SMA, and CTGF to 58\%, 55\%, 42\%, and $65 \%$ of BDL level, respectively.

We demonstrated that baicalin protected against liver fibrosis in a well-established mice of BDL. Baicalin is the glucuronide of baicalein, which is obtained through the binding of glucuronic acid to baicalein. Baicalin has both antioxidant and anti-inflammatory properties [20, 21]. The metabolism of baicalin was studied in rodent model and no toxicity was observed [22, 23]. Liver fibrosis induced by BDL has the deposition of connective tissue elements and formation of ductular followed by necrosis [24]. Role of hepatocytes are crucial in development of liver fibrosis. Cell death of hepatocytes leads to a series of proinflammatory response [25]. Hepatocyte has complex role in liver injuries and multiple factors regulate the process including retinoic acid [26]. It is also known that biliary epithelial cells and hepatocytes can undergo epithelial-mesenchymal transition during liver fibrosis [27]. Expression of profibrotic markers such as collagen I, fibronectin, SMA and CTGF in response to BDL has been reported earlier [5, 18]. We demonstrated that all profibrotic markers were attenuated by alternate day treatment of baicalin. Consistent with our study, the protective effect of baicalin in other mouse models of liver fibrosis has been reported [28].

\subsection{Baicalin Ameliorates BDL-Induced Liver Inflammation in} Mice. BDL induced fibrosis, which is initiated by cell death-associated inflammatory stimuli. We determined 

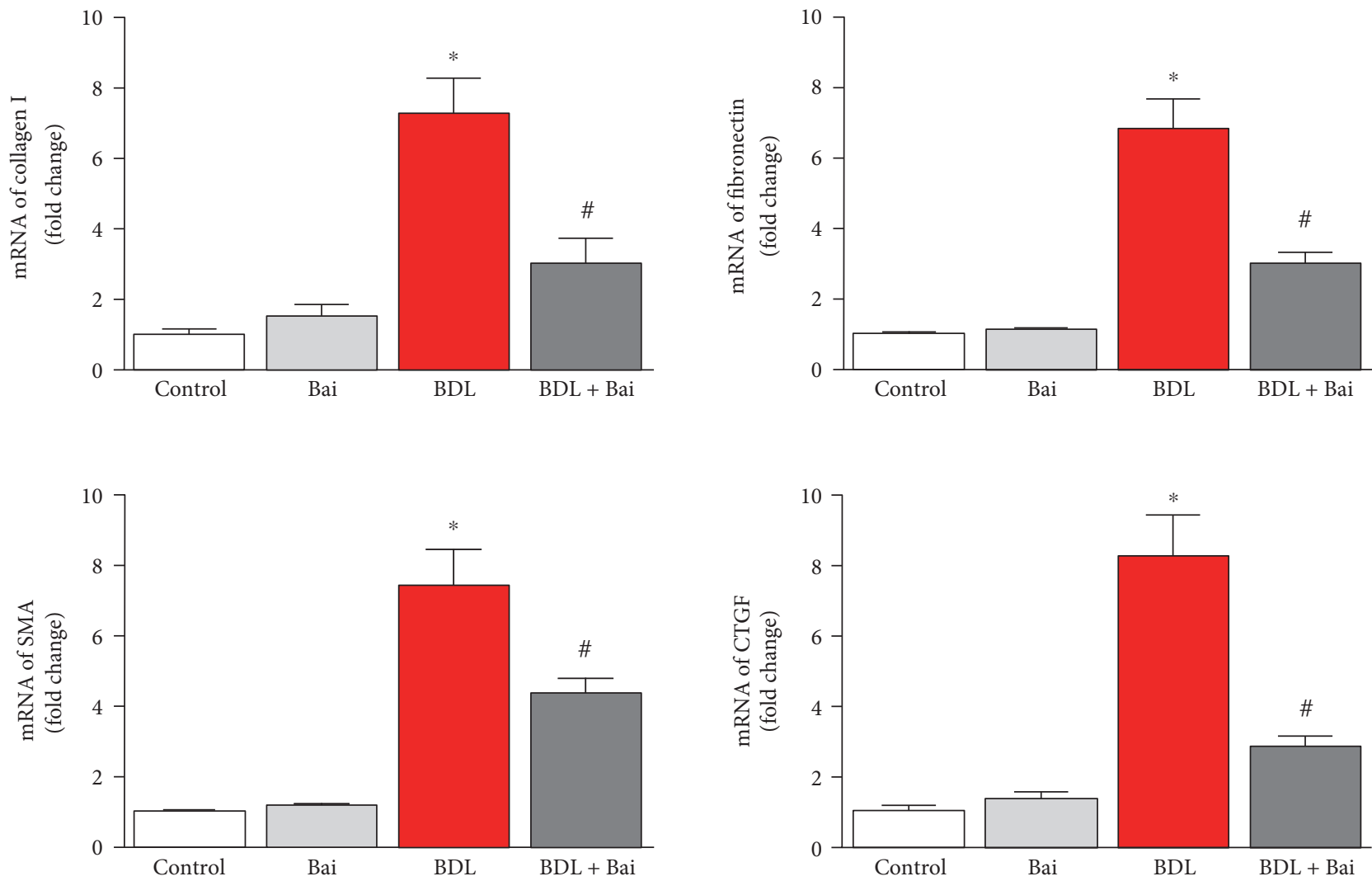

FIGURE 2: Baicalin markedly attenuated BDL-induced fibrotic gene expression. BDL induced liver profibrotic genes at mRNA level. Real-time PCR analyses were performed for collagen I, fibronectin, alpha smooth muscle actin (SMA), and CTGF genes. BDL-induced increases in all four gene expressions and the inductions were significantly reduced by baicalin treatment. Results are mean \pm S.E.M. $n=6$ for each group. ${ }^{*} p<0.05$ versus control and ${ }^{*} p<0.05$ versus BDL.

proinflammatory cytokines TNF $\alpha$, MIP $1 \alpha$, IL $1 \beta$, and MIP2 by real-time PCR. The result demonstrated induced TNF $\alpha$, MIP1 $\alpha$, IL1 $\beta$, and MIP2 mRNA levels to 6.29 -fold, 4.51 -fold, 5.32-fold, and 4.83-fold, respectively (Figure 3(a)). Administration of baicalin on alternate day for 15 days reduced BDLinduced proinflammatory cytokines $\operatorname{TNF} \alpha, \operatorname{MIP} 1 \alpha$, IL $1 \beta$, and MIP2 to $63 \%, 47 \%, 47 \%$, and $49 \%$ respectively. To verify the mRNA signal to protein level, we examined one marker TNF $\alpha$ by immunoblot analyses and BDL increased TNF $\alpha$ protein level and was attenuated by baicalin, which is consistent with the mRNA expression pattern.

Our study demonstrated that baicalin prevents fibrosis by attenuating BDL-induced proinflammatory cytokines and inflammation. Modulation of inflammatory signal by baicalin in the liver for hepato-protection is well known in other liver injuries induced by concanavalin A, D-galactosamine, and alcoholic fatty liver [13, 14, 29]. The resident macrophage, known as the Kupffer cell, plays critical role in this process [30].

\subsection{Baicalin Ameliorates BDL-Induced Oxidative Stress} Markers in Mice. Inflammatory response in BDL induces oxidative stress and reduces cellular antioxidant capability. Here, we determined oxidative/nitrative markers and their causes. HNE protein adducts increased significantly in the liver due to BDL (3.9-fold) and baicalin treatment reduced significantly to $47 \%$ (Figure $4(\mathrm{a})$ ). Hepatic protein nitration is also increased to 3.5 -fold and baicalin attenuated $45.7 \%$ from BDL (Figure 4(a)). The sources of oxidative stress were investigated by real-time PCR of NOX2 and iNOS. Both of them were induced in BDL and attenuated by baicalin. Treatment with baicalin in control group does not have any effect of oxidative stress and ROS-generating sources.

BDL induced significant oxidative stress through bile salt and other free radicals which causes damage to other organs such the kidney in addition to liver [31]. In liver injury, hepatocytes are primary parenchymal cells targeted by oxidative stress [32]. Consistent with our study in BDL, the antioxidant properties of baicalin are reported in many other liver injury models $[28,33]$.

3.4. Baicalin Improves BDL-Impaired Mitochondrial Membrane Complex Activities in Mice. Mitochondrial electron transport chain is one of the major sources for free radical generation and oxidative injury. We examined electron transport chain complex activities from isolated mitochondria of the liver. BDL surgeries in mice reduced complex I, complex II, and complex IV activities to $45 \%, 35 \%$, and $37 \%$, respectively (Figure 5). Administration of baicalin every day during two weeks BDL improved complex I activity to $40 \%$, complex II to $31 \%$, and complex IV to $33 \%$. Thus, baicalin administration significantly ameliorates BDL- 

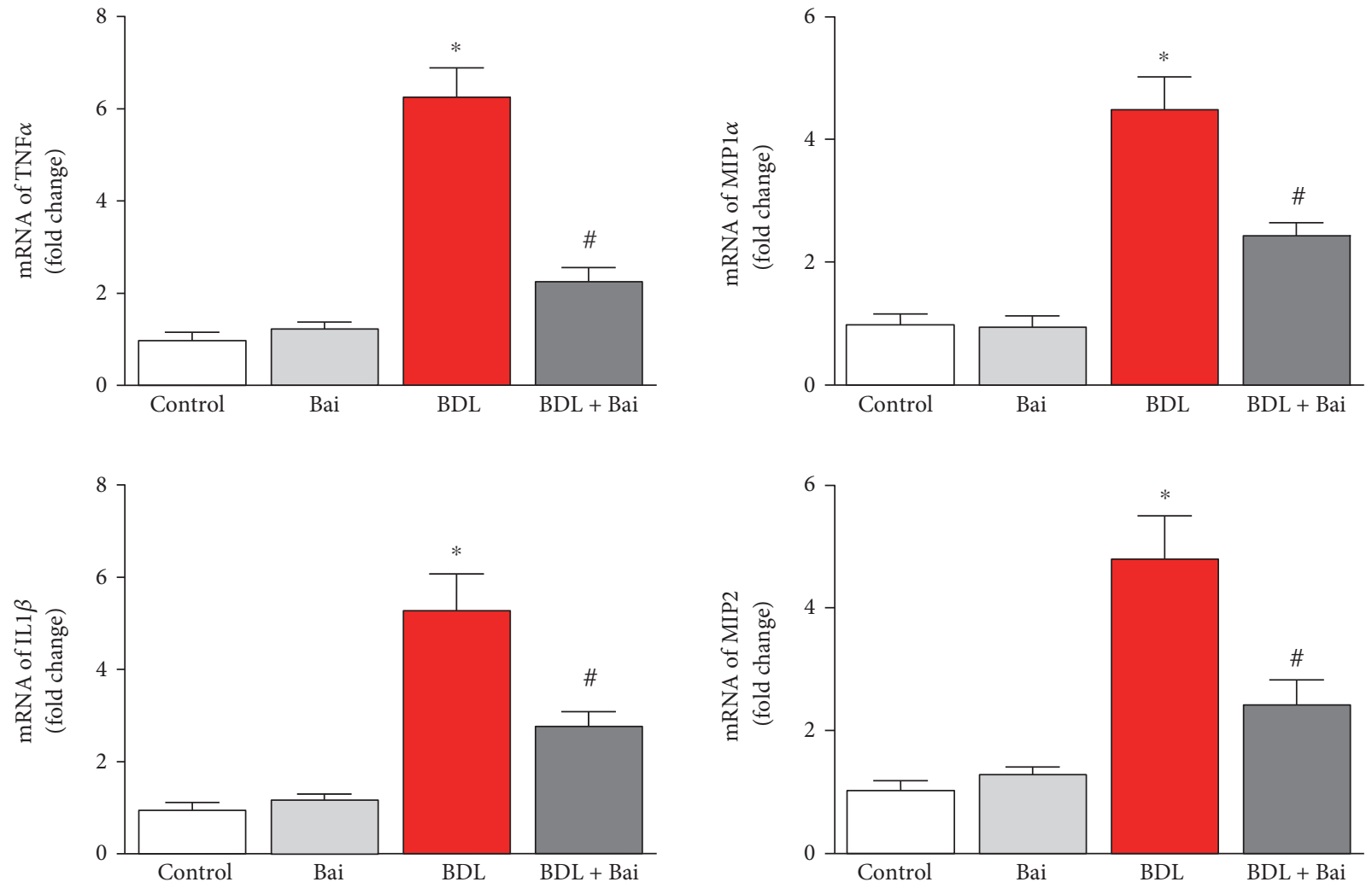

(a)

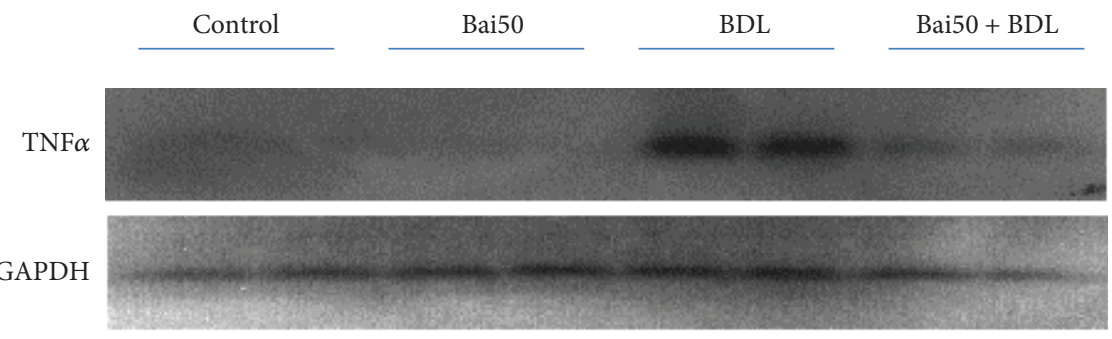

(b)

FIGURE 3: Effect of baicalin on BDL-induced proinflammatory cytokines in mice liver. (a) BDL-induced proinflammatory cytokines as measured by real-time PCR analyses for TNF $\alpha, \operatorname{MIP} 1 \alpha$, IL1 $\beta$, and MIP2 genes. BDL-induced proinflammatory cytokine mRNA was attenuated by alternate day baicalin treatment. Results are mean \pm S.E.M. $n=6$ for each group. ${ }^{*} p<0.05$ versus control and ${ }^{\#} p<0.05$ versus BDL. (b) Protein level of TNF $\alpha$ was verified by immunoblot analyses, and GAPDH was used as the loading control.

induced mitochondrial dysfunction and associated oxidative injury in the liver.

Mitochondria play a bigger role in BDL-induced liver injury $[34,35]$. The dysfunctional electron transport chain in mitochondria is one of the major sources of ROS [36]. We observed dysfunctional electron transport chain in BDL mouse liver, and baicalin improved those functions of electron chain complexes. Baicalin ameliorates mitochondrial damage in streptozotocin-induced diabetic rats, and its beneficial effects on mitochondria are reported [37-39]. Baicalin has a beneficial effect on mitochondrial function [38].

3.5. Baicalin Enhances BDL-Induced Nuclear Translocation of Nrf2 and Increases HO-1 and GCLM Expression. NRF2 is key transcriptional regulator for antioxidant defense in many liver diseases [40]. Baicalin significantly enhanced nuclear localization of NRF2 (Figure 6(a), top panel) in BDL mice. An increase in BDL-induced NRF2 nuclear localization may be due to induction of endogenous antioxidant defense in response to oxidative stress but not sufficient to protect against injury. In addition, we also observed little increase of NRF2 in cytoplasmic fractions of BDL mice pretreated with baicalin (Figure 6(a), bottom panel). These results indicated baicalin mediated upregulation of NRF2 in fibrotic liver. Nuclear translocation of NRF2 is also associated with transcriptional activation as evident by the fact that the target genes (HO-1 and GCLM) also induced in the same pattern as nuclear translocation (Figure 6(b)).

The hepatoprotective effect of many naturally occurring compounds is mediated by NRF2 [41]. NRF2 is also is a 

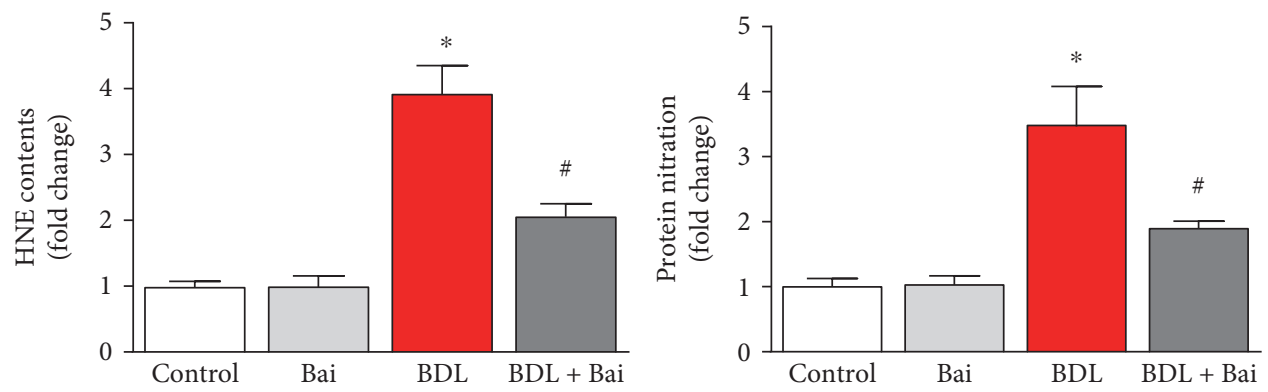

(a)
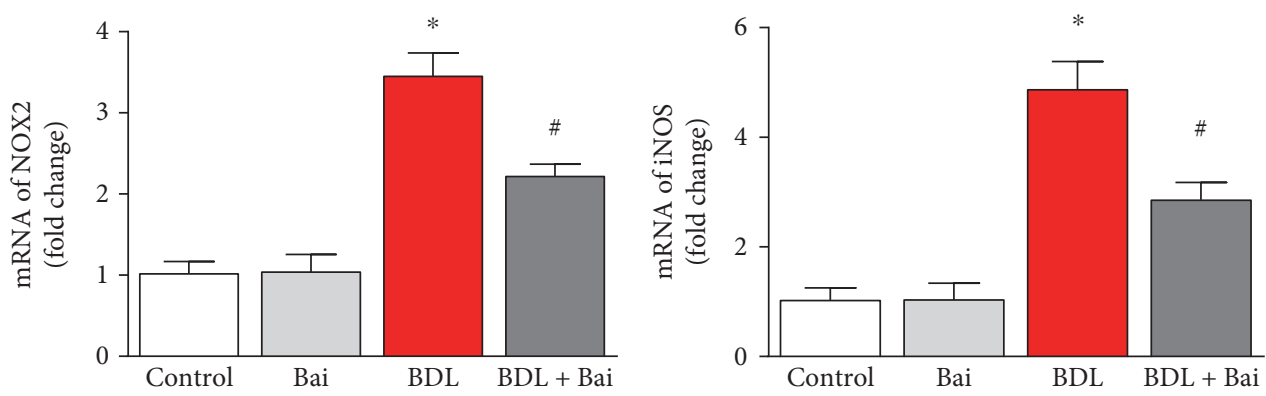

(b)

FIGURE 4: Baicalin significantly reduced BDL-induced oxidative/nitrative stress in mouse liver. BDL caused significant increase in oxidative and nitrative stress as measured by HNE protein adducts and protein nitration using commercial ELISA kits. BDL induced nitrative stress and oxidative stresses (a) and was attenuated by baicalin treatment. Results are mean \pm S.E.M. $n=6$ for each group. ${ }^{*} p<0.05$ versus control and ${ }^{\#} p<0.05$ versus BDL. Real-time PCR analyses were performed for NOX2 and iNOS genes (b). BDL induced both gene expression, and the inductions were significantly reduced by baicalin treatment. Results are mean \pm S.E.M. $n=6$ for each group. ${ }^{*} p<0.05$ versus control and ${ }^{\#} p<0.05$ versus BDL.
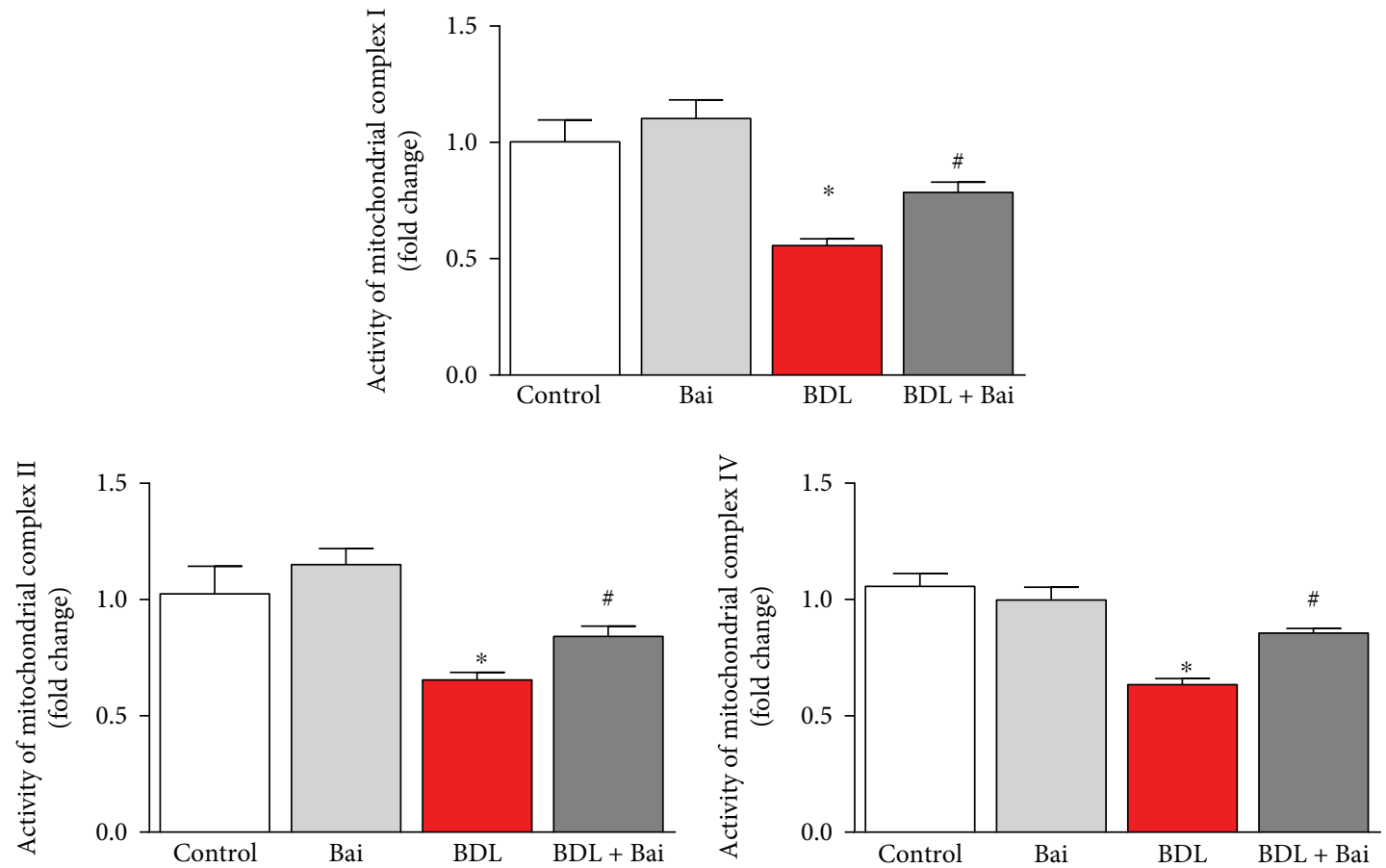

FIGURE 5: Baicalin improved BDL-induced decrease of mitochondrial membrane complex activities in mouse liver. BDL caused decrease in mitochondrial membrane complexes as measured from enzyme activities of electron transport chain complex I, complex II, and complex IV. BDL mediated decrease of mitochondrial complex activities were improved level by alternate day of baicalin treatment. Results are mean \pm S.E.M. $n=6$ for each group. ${ }^{*} p<0.05$ versus control and ${ }^{*} p<0.05$ versus BDL. 


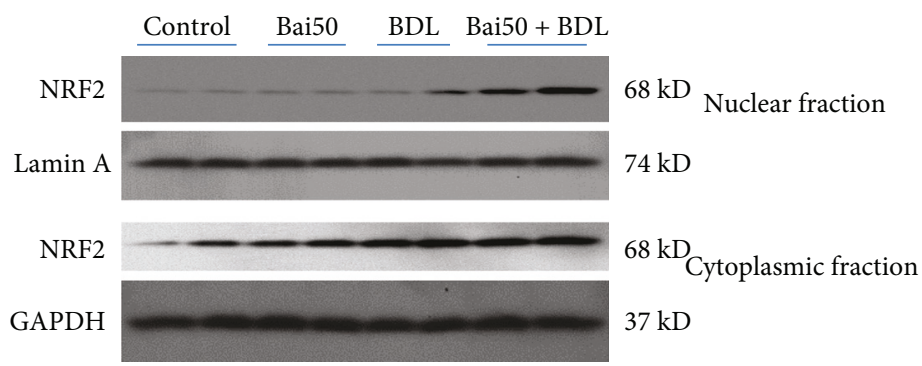

(a)
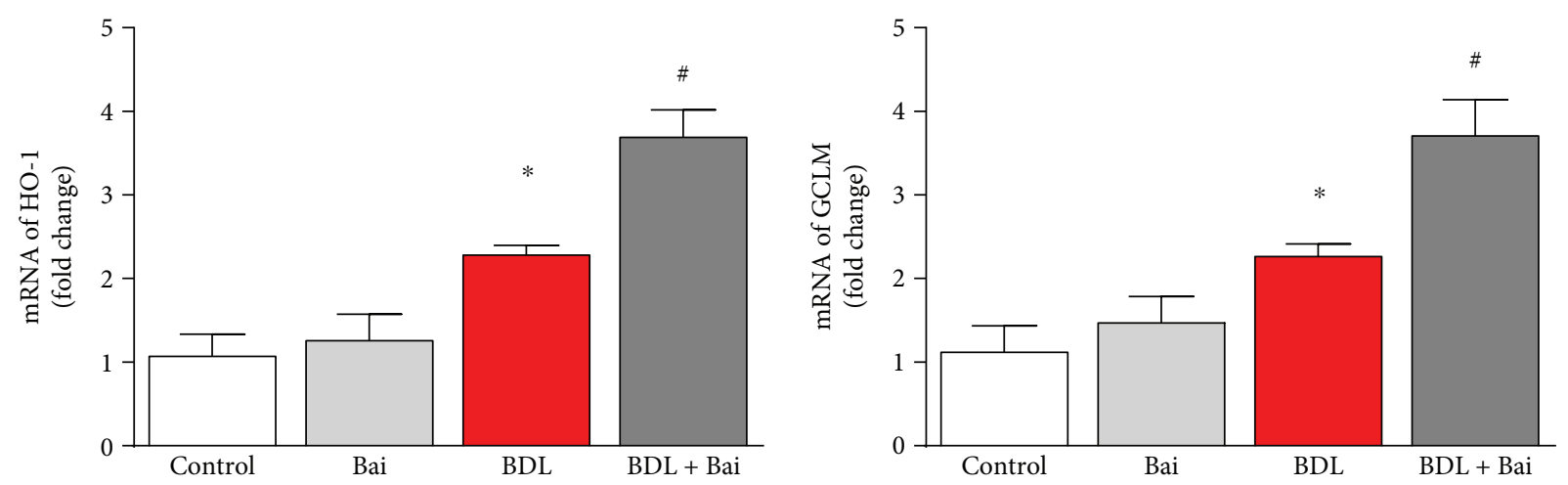

(b)

FIGURE 6: Baicalin enhanced nuclear translocation of NRF2 in BDL mice and increased transcription of its target genes HO-1 and GCLM. (a) Western blot images showing BDL-induced nuclear localization of NRF2 were further enhanced significantly by baicalin treatment. Western blot analyses of cytoplasmic fraction showing increased expression of NRF2 in samples with BDL pretreated with baicalin. (b) Real-time PCR analyses were performed for HO-1 and GCLM genes. BDL induced both gene expressions, and the inductions were significantly enhanced by baicalin treatment. Results are mean \pm S.E.M. $n=6$ for each group. ${ }^{*} p<0.05$ versus control and ${ }^{*} p<0.05$ versus BDL.

key regulator of oxidative stress in numerous cell types $[42,43]$. In BDL, Nrf2-deficient mice show reduced elimination of bile acids leading to liver injury [44]. Consistent with the key role of NRF2 in BDL, we also demonstrated that baicalin modulates NFR2 and its increased nuclear translocation led to protective effect. The induction of antioxidant defense such as $\mathrm{HO}-1$ by baicalin-induced NRF2 translocation may be responsible for hepatoprotection. NRF2 translocation by baicalin is not mediated by any prooxidant properties, but the modulation is at the molecular level. This hypothesis is further supported by the fact that baicalin did not induce NRF2 in the control group.

\subsection{Baicalin Ameliorates BDL-Induced Cell Death Pathway} in Mouse Liver. BDL induces significant apoptotic and necrotic cell death in liver [5]. We examined three cell death markers DNA fragmentation, PARP activity, and caspase 3 activity. BDL induced 3.6-fold and 3.7-fold increase in apoptotic cell death markers DNA fragmentation and caspase 3 activity. Baicalin administration reduced BDLinduced DNA fragmentation and caspase 3 activity up to $40 \%$ and $42 \%$, respectively (Figure $7(\mathrm{a})$ ). We also investigated another cell death marker PARP activity which represents necrotic pathway and apoptotic pathways in liver injury [6]. BDL induced 3-fold increase in PARP activity, and baicalin administration significantly reduced BDL-induced PARP activity (44.5\%) (Figure 7(b)).
Oxidative stress is major source for cell death in BDL $[45,46]$. Intervention of PARP is shown to ameliorate liver fibrosis in BDL [4]. Baicalin prevented BDL-induced apoptotic and necrotic cell death. The effect may be due to reducing oxidative stress, promoting antioxidant defense, and attenuating inflammation.

\subsection{Mechanistic Role of Stellate Cells in Baicalin-Mediated} Antifibrotic Action. Hepatic stellate cells contribute to liver fibrosis through various steps including extracellular matrix homeostasis [47]. As we observed significant drop in liver fibrosis by baicalin, an in vitro study with stellate cells was essential. We used a natural phenomenon where spontaneous activation occurs at day 8 when stellate cells grow under in vitro condition [5]. To elucidate the inhibitory role of baicalin in the activation of stellate cell process and its effect on profibrotic marker production, we examined using real-time PCR analyses. The data demonstrated that baicalin treatment at $20 \mu \mathrm{M}$ significantly reduced profibrotic genes such as SMA, collagen 1, and fibronectin (Figure 8).

After liver injury, stellate cell activation plays central role in initiation and propagation of liver fibrosis [48]. Kupffer cell activation facilitates stellate cell activation by secretion of TGF $\beta$ and ROS in the extracellular environment [30]. We observed modulation of stellate cell activation by baicalin in vitro. This is significant because of the fact that baicalin 

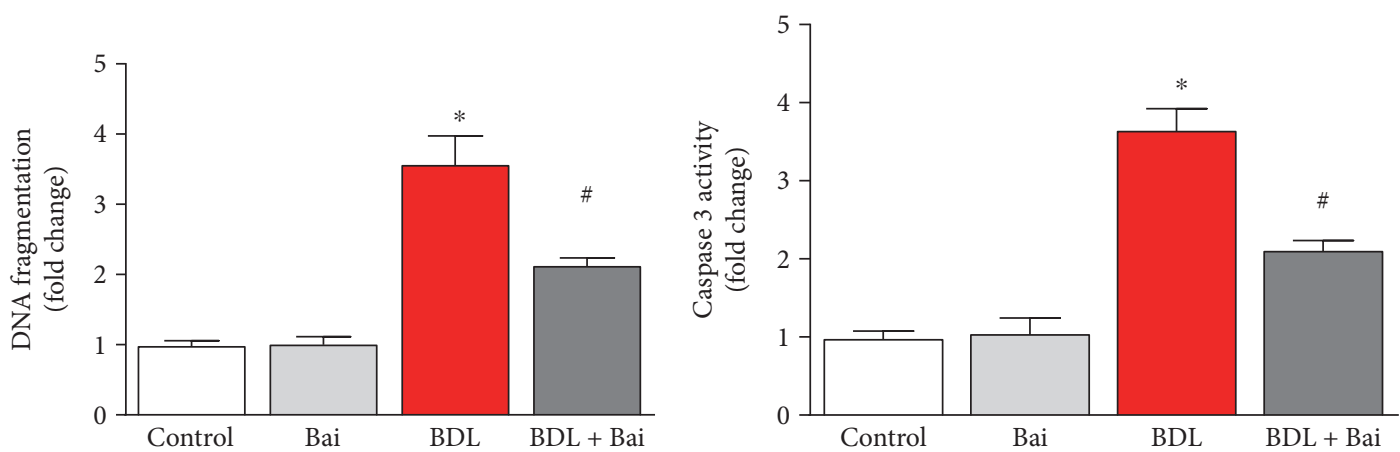

(a)

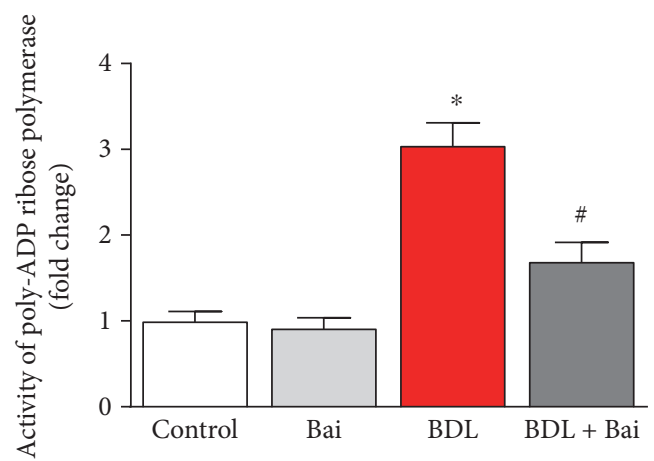

(b)

FIGURE 7: Baicalin markedly attenuated BDL-induced apoptotic and necrotic cell death markers DNA fragmentation, caspase 3 activity, and PARP activity in mouse liver. BDL induced significant increases in liver DNA fragmentation and caspase 3 activity, both markers of apoptotic cell death (a). BDL induced significant increase in PARP activity, a marker of necrotic and apoptotic cell death (b). BDL-induced increases in all three cell death markers were attenuated by baicalin treatment. Results are mean \pm S.E.M. $n=6 /$ group. ${ }^{*} p<0.05$ versus control and ${ }^{\#} p<0.05$ versus BDL.
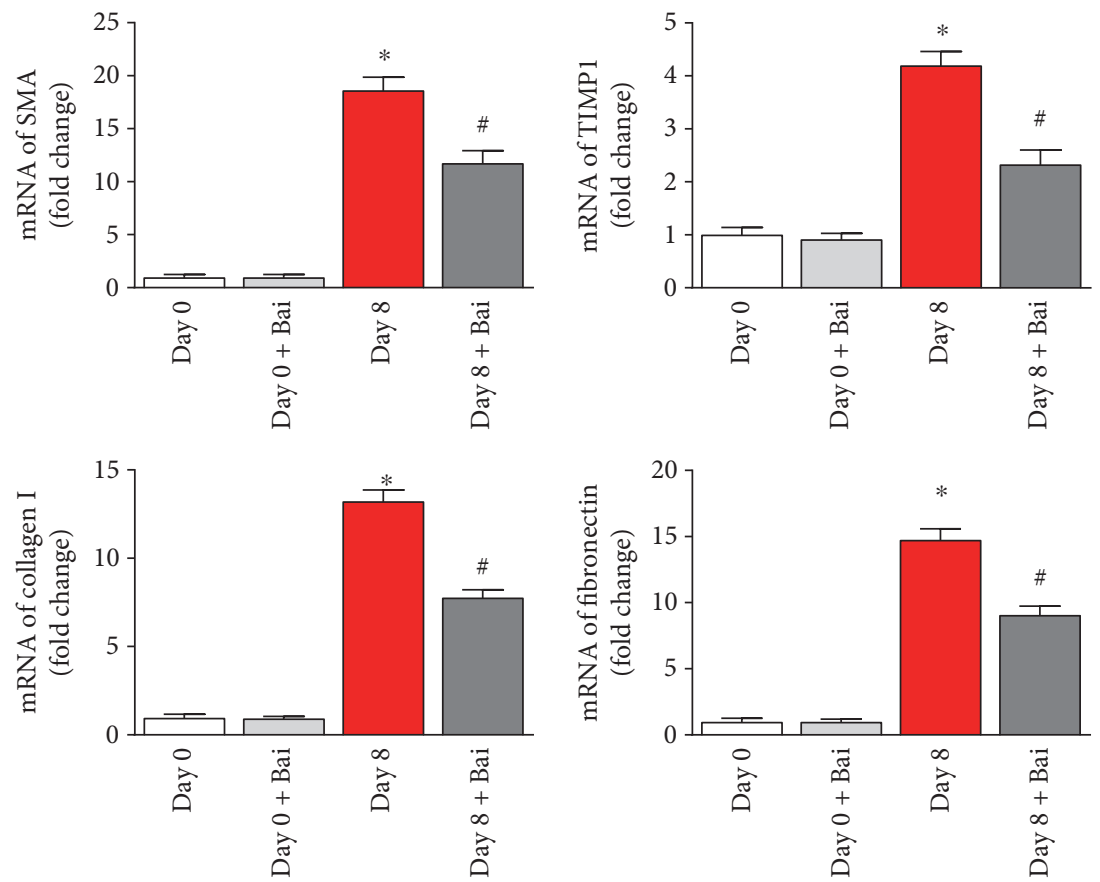

FIGURE 8: Baicalin attenuated profibrotic gene expression in isolated mouse stellate cells. Stellates were cultured for 8 days to stimulate activation in culture and compared with fresh isolated stellate cells (day 0). Real-time PCR analyses of profibrotic mRNA of smooth muscle actin alpha (SMA), TIMP1, collagen 1, and fibronectin were induced during fibrosis stimulation. Baicalin reversed mRNA expressions in all four genes. Results are mean \pm S.E.M. $n=4$ for each group. ${ }^{*} p<0.05$ versus day 0 and ${ }^{\#} p<0.05$ versus day 8 . 


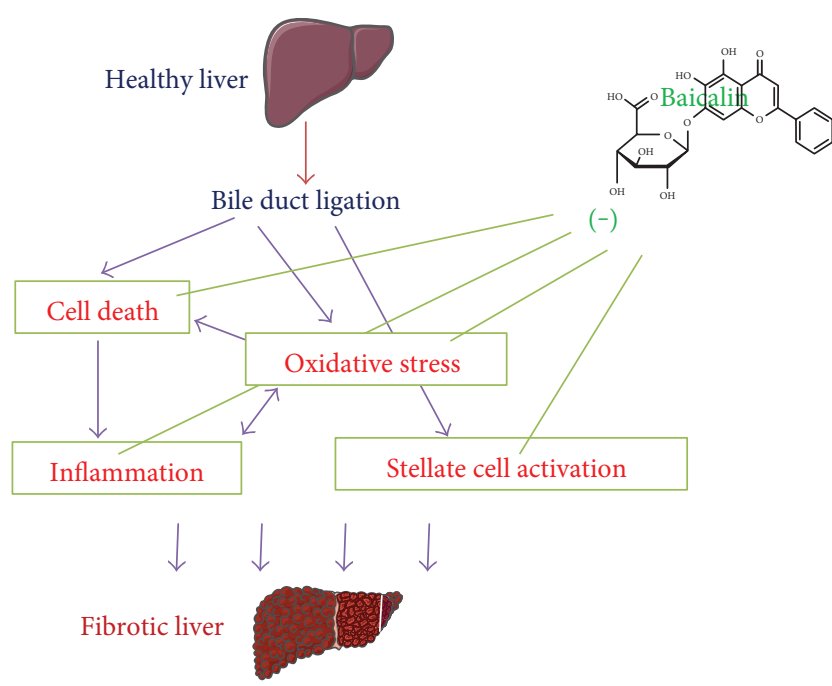

FIgURE 9: Schematic diagram of baicalin-mediated protection in experimental cholestatic liver injury. Bile duct ligation of healthy liver leads to hepatocyte cell death and oxidative stress. Both of these factors lead to proinflammatory responses, and significant inflammation occurs. Inflammation also leads to increased oxidative stress and thus interplay each other. Baicalin protects against cell death, oxidative stress, and inflammation. In addition to that, stellate cell activation occurs which leads to liver fibrosis. Under in vitro condition, baicalin attenuates stellate cell activation. All the above pathophysiological conditions lead to fibrotic liver.

can modulate liver fibrosis not only by reducing oxidative stress/inflammation/cell death but also by regulating stellate cell activation.

\section{Conclusion}

The complexities of liver fibrosis and lack of approved treatment to patients lead this subject to forefront to researchers and clinicians. In our study, baicalin partially protects against experimental liver cholestasis via multiple mechanisms (Figure 9). The complexities of cholestatic liver fibrosis are due to the involvement of different cells in the liver such as hepatocytes, stellate cells, and immune cells, and their interactions are real crucial. Reversal of such process is challenging. Thus, the limitation of baicalin-mediated protection is due to the additional challenging factors including bioavailability, selective transportation to hepatocytes or stellate cells, or targeting other cell types. Despite the above limitations, baicalin protects against experimental cholestatic liver fibrosis by reducing inflammation, oxidative stress, and cell death and enhancing antioxidant defense in addition to modulating stellate cell activation.

\section{Conflicts of Interest}

The authors declare that there is no conflict of interest regarding the publication of this paper.

\section{Authors' Contributions}

Kezhen Shen and Xiaowen Feng contributed equally to this work.

\section{Acknowledgments}

This work was supported by grants from the Major Program of National Natural Science Foundation of China (no. 91542205), Science and Technology Department of Zhejiang Province (no. 2015C33214), and Zhejiang Medical and Health Science and Technology Plan Project (nos. 2014KYA070 and 200815385).

\section{References}

[1] Y. S. Lim and W. R. Kim, "The global impact of hepatic fibrosis and end-stage liver disease," Clinics in Liver Disease, vol. 12, pp. 733-746, 2008, vii.

[2] T. E. Starzl, A. J. Demetris, and D. Van Thiel, "Liver transplantation (1)," The New England Journal of Medicine, vol. 321, pp. 1014-1022, 1989.

[3] M. A. Doyle, J. Singer, T. Lee, M. Muir, and C. Cooper, "Improving treatment and liver fibrosis outcomes with metformin in HCV-HIV co-infected and HCV mono-infected patients with insulin resistance: study protocol for a randomized controlled trial," Trials, vol. 17, p. 331, 2016.

[4] Y. Koyama, J. Xu, X. Liu, and D. A. Brenner, "New developments on the treatment of liver fibrosis," Digestive Diseases, vol. 34, pp. 589-596, 2016.

[5] K. Shen, X. Feng, R. Su, H. Xie, L. Zhou, and S. Zheng, "Epigallocatechin 3-gallate ameliorates bile duct ligation induced liver injury in mice by modulation of mitochondrial oxidative stress and inflammation," PloS One, vol. 10, article e0126278, 2015.

[6] P. Mukhopadhyay, M. Rajesh, Z. Cao et al., "Poly (ADPribose) polymerase-1 is a key mediator of liver inflammation and fibrosis," Hepatology, vol. 59, pp. 1998-2009, 2014.

[7] T. Kisseleva and D. A. Brenner, "Hepatic stellate cells and the reversal of fibrosis," Journal of Gastroenterology and Hepatology, vol. 21, Supplement 3, pp. S84-S87, 2006.

[8] A. Canbay, P. Taimr, N. Torok, H. Higuchi, S. Friedman, and G. J. Gores, "Apoptotic body engulfment by a human stellate cell line is profibrogenic," Laboratory Investigation, vol. 83, pp. 655-663, 2003.

[9] A. Canbay, A. E. Feldstein, H. Higuchi et al., "Kupffer cell engulfment of apoptotic bodies stimulates death ligand and cytokine expression," Hepatology, vol. 38, pp. 11881198, 2003.

[10] A. L. Harvey, R. Edrada-Ebel, and R. J. Quinn, "The reemergence of natural products for drug discovery in the genomics era," Nature Reviews Drug Discovery, vol. 14, pp. 111-129, 2015.

[11] D. Wozniak, E. Lamer-Zarawska, and A. Matkowski, "Antimutagenic and antiradical properties of flavones from the roots of Scutellaria baicalensis georgi," Die Nahrung, vol. 48, pp. 9-12, 2004.

[12] S. J. Kim, Y. J. Moon, and S. M. Lee, "Protective effects of baicalin against ischemia/reperfusion injury in rat liver," Journal of Natural Products, vol. 73, pp. 2003-2008, 2010. 
[13] L. L. Liu, L. K. Gong, H. Wang et al., "Baicalin protects mouse from concanavalin A-induced liver injury through inhibition of cytokine production and hepatocyte apoptosis," Liver International, vol. 27, pp. 582-591, 2007.

[14] H. Wang, Y. Zhang, R. Bai, M. Wang, and S. Du, "Baicalin attenuates alcoholic liver injury through modulation of hepatic oxidative stress, inflammation and sonic hedgehog pathway in rats," Cellular Physiology and Biochemistry, vol. 39, pp. 11291140, 2016.

[15] Y. L. Xi, H. X. Li, C. Chen et al., "Baicalin attenuates high fat diet-induced insulin resistance and ectopic fat storage in skeletal muscle, through modulating the protein kinase B/glycogen synthase kinase 3 beta pathway," Chinese Journal of Natural Medicines, vol. 14, pp. 48-55, 2016.

[16] Y. Xi, M. Wu, H. Li et al., "Baicalin attenuates high fat diet-induced obesity and liver dysfunction: dose-response and potential role of CaMKKbeta/AMPK/ACC pathway," Cellular Physiology and Biochemistry, vol. 35, pp. 23492359, 2015.

[17] H. Qiao, H. Han, D. Hong, Z. Ren, Y. Chen, and C. Zhou, "Protective effects of baicalin on carbon tetrachloride induced liver injury by activating PPARgamma and inhibiting TGFbetal," Pharmaceutical Biology, vol. 49, pp. 38-45, 2011.

[18] F. Shi, Q. Sheng, X. Xu, W. Huang, and Y. J. Kang, "Zinc supplementation suppresses the progression of bile duct ligation-induced liver fibrosis in mice," Experimental Biology and Medicine, vol. 240, pp. 1197-1204, 2015.

[19] H. Pan, J. Chen, K. Shen et al., "Mitochondrial modulation by epigallocatechin 3-gallate ameliorates cisplatin induced renal injury through decreasing oxidative/nitrative stress, inflammation and NF-kB in mice," PloS One, vol. 10, article e0124775, 2015.

[20] D. E. Shieh, L. T. Liu, and C. C. Lin, "Antioxidant and free radical scavenging effects of baicalein, baicalin and wogonin," Anticancer Research, vol. 20, pp. 2861-2865, 2000.

[21] B. Q. Li, T. Fu, W. H. Gong et al., "The flavonoid baicalin exhibits anti-inflammatory activity by binding to chemokines," Immunopharmacology, vol. 49, pp. 295-306, 2000.

[22] Y. Wang, J. Yang, X. Li, and J. Wang, "The metabolism of baicalin in rat and the biological activities of the metabolites," Evidence-Based Complementary and Alternative Medicine, vol. 2012, Article ID 404529, 6 pages, 2012.

[23] M. Y. Lai, S. L. Hsiu, S. Y. Tsai, Y. C. Hou, and P. D. Chao, "Comparison of metabolic pharmacokinetics of baicalin and baicalein in rats," The Journal of Pharmacy and Pharmacology, vol. 55, pp. 205-209, 2003.

[24] S. Hinz, H. Franke, G. Machnik, A. Muller, and R. Dargel, "Histological and biochemical changes induced by total bile duct ligation in the rat," Experimental and Toxicologic Pathology, vol. 49, pp. 281-288, 1997.

[25] H. Malhi, M. E. Guicciardi, and G. J. Gores, "Hepatocyte death: a clear and present danger," Physiological Reviews, vol. 90, pp. 1165-1194, 2010

[26] B. Mukhopadhyay, J. Liu, D. Osei-Hyiaman et al., "Transcriptional regulation of cannabinoid receptor-1 expression in the liver by retinoic acid acting via retinoic acid receptor-gamma," The Journal of Biological Chemistry, vol. 285, pp. 19002-19011, 2010.
[27] M. D. Gorrell, "Liver fibrosis: the hepatocyte revisited," Нераtology, vol. 46, pp. 1659-1661, 2007.

[28] S. W. Park, C. H. Lee, Y. S. Kim et al., "Protective effect of baicalin against carbon tetrachloride-induced acute hepatic injury in mice," Journal of Pharmacological Sciences, vol. 106, pp. 136-143, 2008.

[29] L. L. Liu, L. K. Gong, H. Wang et al., "Baicalin inhibits macrophage activation by lipopolysaccharide and protects mice from endotoxin shock," Biochemical Pharmacology, vol. 75, pp. 914-922, 2008.

[30] G. Kolios, V. Valatas, and E. Kouroumalis, "Role of Kupffer cells in the pathogenesis of liver disease," World Journal of Gastroenterology, vol. 12, pp. 7413-7420, 2006.

[31] M. Orellana, R. Rodrigo, L. Thielemann, and V. Guajardo, "Bile duct ligation and oxidative stress in the rat: effects in liver and kidney," Comparative Biochemistry and Physiology Part C: Pharmacology, Toxicology and Endocrinology, vol. 126, pp. 105-111, 2000.

[32] J. B. Hoek and J. G. Pastorino, "Ethanol, oxidative stress, and cytokine-induced liver cell injury," Alcohol, vol. 27, pp. 63-68, 2002.

[33] C. C. Liao, Y. J. Day, H. C. Lee, J. T. Liou, A. H. Chou, and F. C. Liu, "Baicalin attenuates IL-17-mediated acetaminopheninduced liver injury in a mouse model," PloS One, vol. 11, article e0166856, 2016.

[34] M. M. Tiao, T. K. Lin, P. W. Wang, J. B. Chen, and C. W. Liou, "The role of mitochondria in cholestatic liver injury," Chang Gung Medical Journal, vol. 32, pp. 346-353, 2009.

[35] N. Alptekin, G. Mehmetcik, M. Uysal, and G. Aykac-toker, "Evidence for oxidative stress in the hepatic mitochondria of bile duct ligated rats," Pharmacological Research, vol. 36, pp. 243-247, 1997.

[36] K. M. Holmstrom and T. Finkel, "Cellular mechanisms and physiological consequences of redox-dependent signalling," Nature Reviews Molecular Cell Biology, vol. 15, pp. 411-421, 2014.

[37] V. Y. Waisundara, A. Hsu, B. K. Tan, and D. Huang, "Baicalin reduces mitochondrial damage in streptozotocin-induced diabetic Wistar rats," Diabetes/Metabolism Research and Reviews, vol. 25, pp. 671-677, 2009.

[38] M. R. de Oliveira, S. F. Nabavi, S. Habtemariam, I. Erdogan Orhan, M. Daglia, and S. M. Nabavi, "The effects of baicalein and baicalin on mitochondrial function and dynamics: a review," Pharmacological Research, vol. 100, pp. 296-308, 2015.

[39] W. H. Chang, C. H. Chen, and F. J. Lu, "Different effects of baicalein, baicalin and wogonin on mitochondrial function, glutathione content and cell cycle progression in human hepatoma cell lines," Planta Medica, vol. 68, pp. 128-132, 2002.

[40] S. M. Shin, J. H. Yang, and S. H. Ki, "Role of the Nrf2-ARE pathway in liver diseases," Oxidative Medicine and Cellular Longevity, vol. 2013, Article ID 763257, 9 pages, 2013.

[41] R. N. Jadeja, K. K. Upadhyay, R. V. Devkar, and S. Khurana, "Naturally occurring Nrf2 activators: potential in treatment of liver injury," Oxidative Medicine and Cellular Longevity, vol. 2016, Article ID 3453926, 13 pages, 2016.

[42] H. Zhang, K. J. Davies, and H. J. Forman, "Oxidative stress response and Nrf2 signaling in aging," Free Radical Biology \& Medicine, vol. 88, pp. 314-336, 2015.

[43] O. Al-Sawaf, T. Clarner, A. Fragoulis et al., "Nrf2 in health and disease: current and future clinical implications," Clinical Science, vol. 129, pp. 989-999, 2015. 
[44] L. M. Aleksunes, A. L. Slitt, J. M. Maher et al., "Nuclear factorE2-related factor 2 expression in liver is critical for induction of NAD(P)H:quinone oxidoreductase 1 during cholestasis," Cell Stress \& Chaperones, vol. 11, pp. 356-363, 2006.

[45] F. J. Padillo, A. Cruz, C. Navarrete et al., "Melatonin prevents oxidative stress and hepatocyte cell death induced by experimental cholestasis," Free Radical Research, vol. 38, pp. 697-704, 2004.

[46] C. Ara, H. Kirimlioglu, A. B. Karabulut et al., "Protective effect of resveratrol against oxidative stress in cholestasis," The Journal of Surgical Research, vol. 127, pp. 112-117, 2005.

[47] J. E. Puche, Y. Saiman, and S. L. Friedman, "Hepatic stellate cells and liver fibrosis," Comprehensive Physiology, vol. 3, pp. 1473-1492, 2013.

[48] M. J. Arthur, D. A. Mann, and J. P. Iredale, "Tissue inhibitors of metalloproteinases, hepatic stellate cells and liver fibrosis," Journal of Gastroenterology and Hepatology, vol. 13, pp. S33S38, 1998. 


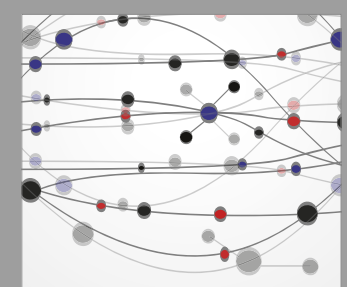

The Scientific World Journal
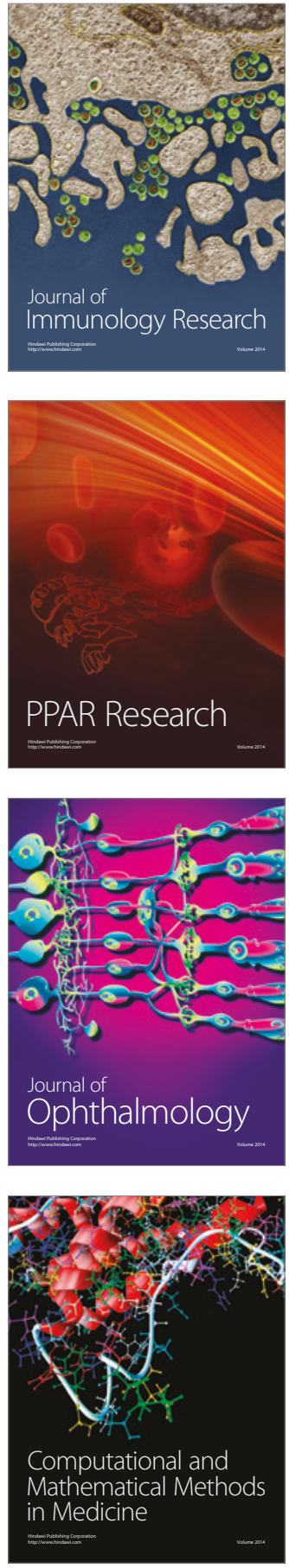

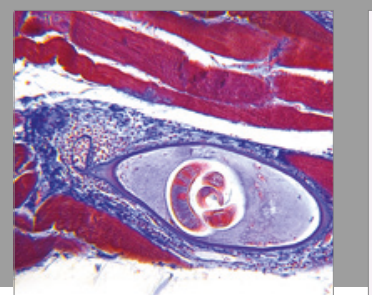

Gastroenterology Research and Practice
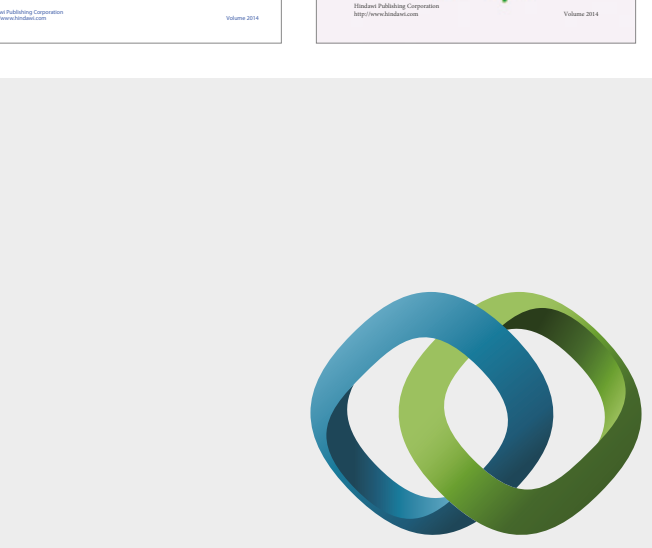

\section{Hindawi}

Submit your manuscripts at

https://www.hindawi.com
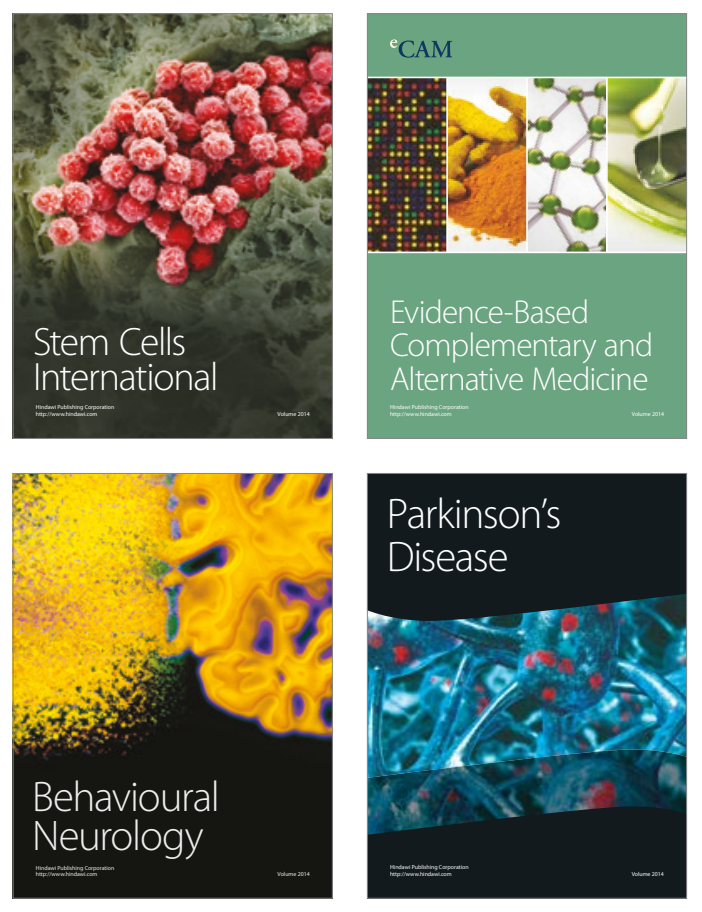
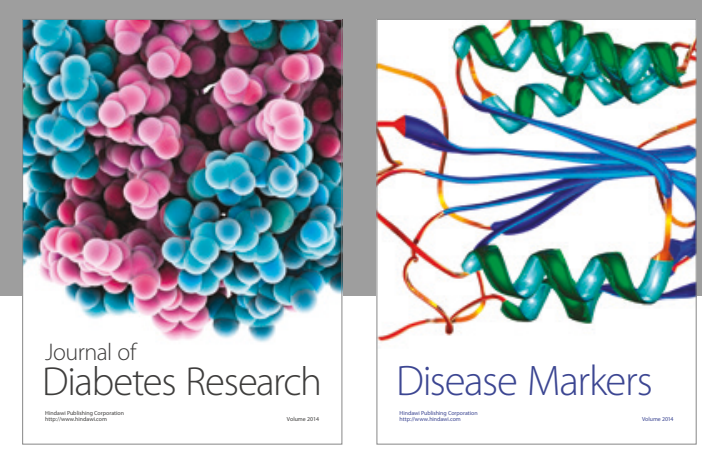

Disease Markers
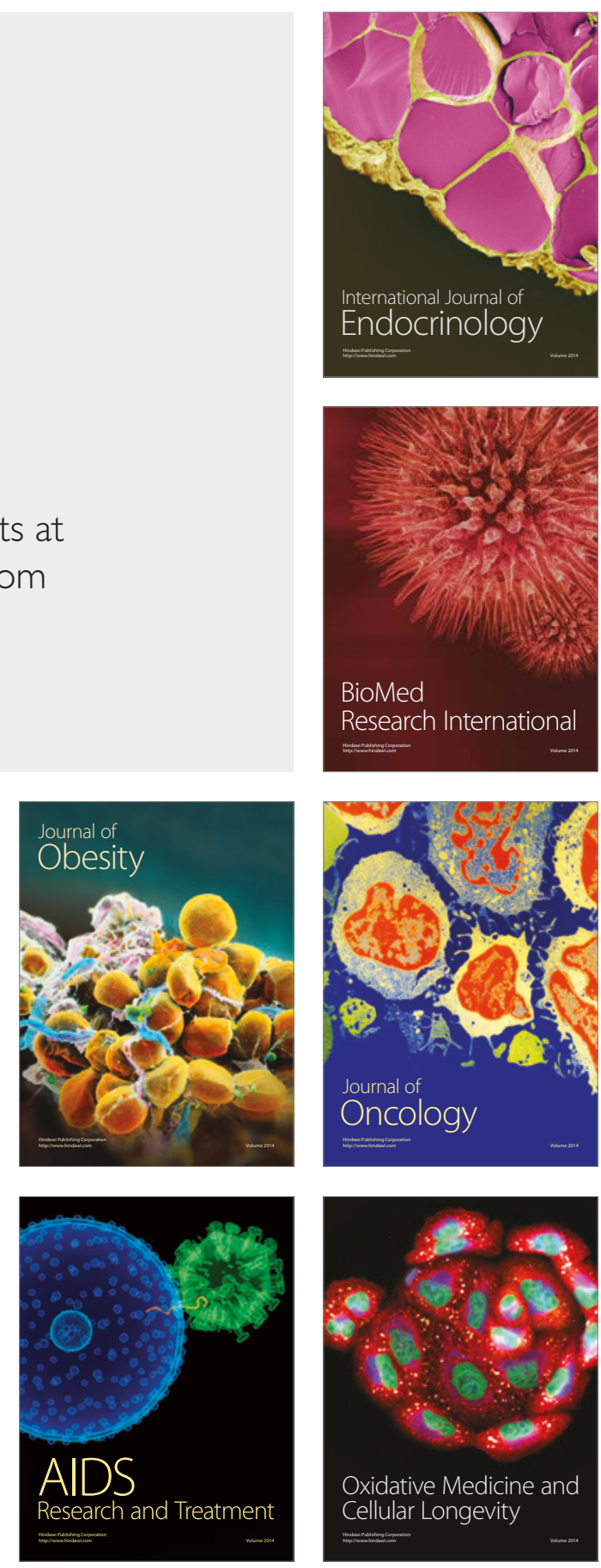